\title{
A Inovação e a Indústria 4.0: Proposta para utilização de elementos para uma organização competitiva
}

\author{
The Innovation and Industry 4.0: Proposal for the use of elements for a competitive organization \\ Innovación e a Industria 4.0: propuesta de uso de elementos para una organización competitiva
}

Recebido: 29/06/2021 | Revisado: 06/07/2021 | Aceito: 07/07/2021 | Publicado: 09/07/2021

\author{
Marcos de Oliveira Morais \\ ORCID: https://orcid.org/0000-0002-5981-4725 \\ Universidade Estácio de Sá, Brasil \\ Universidade Santo Amaro, Brasil \\ E-mail: marcostecnologia2001@gmail.com \\ Diogo Fernando Maria \\ ORCID: https://orcid.org/0000-0002-6912-5085 \\ Universidade Estácio de Sá, Brasil \\ E-mail: diogofernandobranco1@gmail.com \\ Leandro Marcelino de Oliveira \\ ORCID: https://orcid.org/0000-0001-9632-3178 \\ Universidade Estácio de Sá, Brasil \\ E-mail: marcelino.leandro@yahoo.com.br
}

\begin{abstract}
Resumo
O presente estudo tem a proposta de refletir acerca dos desafios e possibilidades que o processo e inovação e da indústria 4.0 podem potencializar as ações organizacionais em ambientes corporativos frente às novas necessidades de mercado. A metodologia se dará por meio de levantamento literário nos assuntos em foco agregando valor a discussão. Também é proposto um modelo para ser um norteador quanto a obtenção de vantagem competitiva. Nesse sentido os autores pretendem fomentar o assunto sem a pretensão de estanca-lo e sim de modo contribuir com a literatura na expansão do conhecimento e na troca de informações agregando valor para as organizações, auxiliando no gerenciamento organizacional bem como para a literatura especializada.
\end{abstract}

Palavras-chave: Inovação; Cultura 4.0; Crescimento organizacional; Estratégia organizacional.

\begin{abstract}
This study aims to reflect on the challenges and possibilities that the process and innovation and industry 4.0 can enhance organizational actions in corporate environments in view of the new market needs. The methodology will be through a literary survey on the subjects in focus, adding value to the discussion. A model is also proposed to guide the achievement of competitive advantage. In this sense, the authors intend to promote the subject without intending to stop it, but in order to contribute to the literature in the expansion of knowledge and in the exchange of information, adding value to organizations, assisting in organizational management as well as in specialized literature.
\end{abstract}

Keywords: Innovation; Culture 4.0; Organizational growth; Organizational strategy.

\section{Resumen}

Este estudio tiene como objetivo reflexionar sobre los desafíos y posibilidades que el proceso y la innovación y la industria 4.0 pueden potenciar las acciones organizacionales en entornos corporativos ante las nuevas necesidades del mercado. La metodología será a través de una encuesta literaria sobre los temas en foco, agregando valor a la discusión. También se propone un modelo para orientar el logro de la ventaja competitiva. En este sentido, los autores pretenden promover el tema sin pretender detenerlo, pero con el fin de contribuir a la literatura en la expansión del conocimiento y en el intercambio de información, agregando valor a las organizaciones, asistiendo en la gestión organizacional así como en literatura especializada.

Palabras clave: Innovación; Cultura 4.0; Crecimiento organizacional; Estrategia organizacional.

\section{Introdução}

A busca pelo processo de inovação nas organizações permite desenvolver técnicas de gestão com o intuito de promover e aumentar a produtividade bem como a eficiência e eficácia organizacional objetivando um melhor posicionamento no mercado em que a empresa atua, tornando-a mais competitiva e lucrativa. 
A inovação aliada ao conhecimento tornou-se um fator vital para a sobrevivência das organizações, atribuindo-se a ela o sucesso organizacional e o crescimento tida como condutor principal do desenvolvimento econômico possibilitando também favorecer no crescimento econômico do país (Low; Kalafut, 2003; Santos et al, 2014).

A primissa da Indústria 4.0, envolvem elementos da manufatura inteligente aplicada aos diversos setores das organizações, onde a tecnologias da informação e comunicação (TIC), tecnologia industrial e depende, principalmente, da construção de sistemas Cyber-físicos (CPS) para criar uma fábrica digital e inteligente, a fim de tornar a fabricação mais orientada a informações, de modo personalizado e limpo. O foco da Indústria 4.0 esta em construir um modelo de produção flexível de produtos, processos e/ou serviços cada vez mais personalizados e digitais, com interações em tempo real entre Homem e máquinas, produtos e dispositivos durante o processo produtivo (Zhou, Liu, \& Zhou, 2016).

Atuar na busca por melhores resultados e em meio as novas oportunidades e tecnologia permite que seja possível fomentar novas maneiras de gestão, sejam estas organizacionais ou mesmo tecnológicas. Rubmann et al. (2015) já afirmavam que o surgimento de novas tecnologias industriais, denominadas de Indústria 4.0, auxiliada por tecnologias digitais, tais como os CPS, a IoT, o Big Data e a computação em nuvem ou Cloud Computing (CC), iriam exercer transformações que aumentariam os níveis de produtividade das fábricas, promovendo o crescimento industrial, modificando o perfil da força de trabalho e alterando também a competitividade das empresas e regiões.

Aliar e potencializar os avanços da Internet, IoT, Big Data, computação em nuvem, inteligência artificial e outras TI de nova geração, têm trazido oportunidades valiosas para muitos setores da indústria, dos serviços e também relacionado aos processos de um modo geral (Qi \& Tao, 2018).

O objetivo do presente artigo esta em ampliar a discussão sobre o tema que embora relativamente novo, torna-se de extrema relevância e possibilita uma ampla dissiminação do conhecimento bem como a utilização de ferramentas ainda não tão exploradas por grande parte das organizações, onde em um ambiente dinâmico de rápidas transformações digitais, as organizações necessitam criar vantagem competitiva e as inovações decorrentes das tecnologias aplicadas pela Indústria 4.0 sendo assim um importante elemento integrador para o atingimento deste fim.

\section{Referencial Teórico}

\subsection{Inovação Organizacional}

O processo de inovação pode trazer retornos altamente lucrativos permitindo assim a criação de vantagem competitiva para as empresas. Onde estas inovações não se limitam quanto a máquinas e equipamentos, podendo ser aplicado também ao capital humano empregado na criação destas vatagens, permitindo assim a quebra de possíveis paradígmas facilitando todo o processo da organização tornando viável a busca por novos mercados de atuação e a sua permanência no mercado em que atua com vistas a crescimentos para novas àreas. Uma empresa possui vantagem competitiva, quando é capaz de gerar mais valor econômico do que suas concorrentes (Barney \& Hesterly, 2006).

$\mathrm{Na}$ teoria schumpeteriana a inovação é descrita como novas combinações entre materiais e forças produtivas a fim de viabilizar novos produtos e o desenvolvimento econômico, e esse processo dinâmico de substituição de antigas tecnologias por novas é denominado "destruição criadora" (Schumpeter, 1982).

As inovações organizacionais se tornam mais frequentes após o processo de globalização que permite a criação deste diferencial para as empresas que estão dispostas a enfrentar novos desafios, principalmente na melhoria e criação de produtos, processos e/ou serviços, fomentando assim um crescimentotecnológico para todos os envolvidos neste processo, sejam eles internos ou mesmos esternos. Pode-se dizer ainda, que a inovação atua em rede, atingindo a interação da empresa inovadora com outros sistemas inovadores, sendo eles universidades, fornecedores, clientes e consumidores (OCDE, 2005).

O Manual de Oslo (2005), estudo publicado pela Organização para Cooperação e Desenvolvimento Econômico 
(OCDE) voltado à mensuração e interpretação de dados relacionados às atividades de Ciência, Tecnologia e Inovação (CT\&I), define quatro tipos de inovação que incluem produtos ou métodos novos ou significativamente melhorados, conforme o Quadro 1:

Quadro 1: Tipos de Inovação.

\begin{tabular}{|c|l|}
\hline $\begin{array}{c}\text { Tipos de } \\
\text { Inovação }\end{array}$ & Descrição \\
\hline $\begin{array}{c}\text { Inovação de } \\
\text { Produto }\end{array}$ & $\begin{array}{l}\text { Introduçãodeum bem ou serviço, incluindomelhoramentos significativos em } \\
\text { especificaçōes técnicas, componentes e materiais, softwares incorporados, } \\
\text { facilidade de uso ou outras caracteristicas funcionais. }\end{array}$ \\
\hline $\begin{array}{c}\text { Inovação de } \\
\text { Processo }\end{array}$ & $\begin{array}{l}\text { Implementação de um método de produção ou distribuição, incluindo } \\
\text { mudanças significativas em técnicas, equipamentos e/ou softwares } \\
\text { utilizados para produzir bens e serviços. }\end{array}$ \\
\hline $\begin{array}{c}\text { Inovação de } \\
\text { Marketing }\end{array}$ & $\begin{array}{l}\text { Implementação de um novo método de marketing com mudanças } \\
\text { significativas na concepção do produto ou em sua embalagem, no } \\
\text { posicionamento do produto, em sua promoçāo ou na fixação de preços. }\end{array}$ \\
\hline $\begin{array}{c}\text { Inovação } \\
\text { Organizacional }\end{array}$ & $\begin{array}{l}\text { Implementação de um novo método organizacional nas práticas de negócios } \\
\text { da empresa, na organização do seu local de trabalho ou em suas relaçōes } \\
\text { externas. }\end{array}$ \\
\hline
\end{tabular}

Fonte: Adaptado do Manual de Oslo (2005).

Buscar e manter os bons resultados obtidos pelo processo de inovação organizacional torna-se cada vez mais complexos, onde no encontro de toda esta evolução aportam-se as transformações advindas da indústria 4.0 que foi descrito na Alemanha em 2011 e que uniu a fabricação industrial com a tecnologia da informação permitindo com que os processos se tornassem cada vez mais colaborativos acrescentando maior precisão e exatidão as ações domadas no processo decisório (Kamble, Gunasekaran, \& Gawankar, 2018).

As inovações provenientes da automação e das tecnologias inteligentes fazem com que as empresas tenham um ritimo acelerado quanto as mudanças produtivas proporcionando resultados radicais na inovação organizacional proposta pela quarta revolução industrial ou simplesmente indústria 4.0. Essa análise corroboram as afirmações de Hofmann e Rüsch (2017), para quem a evolução e a capacitação das empresas e de seus funcionários é um dos pontos centrais do desenvolvimento da indústria 4.0.

Compreender, entender e assimilar o modo como a inovação se configura no ambiente organizacional a partir de fatores objetivos, estruturais e também subjetivos foi, então, o primeiro passo para a identificação do caráter multifacetado do fenômeno relacionado a indústria 4.0 (Santos; Basso; Kimura, 2012).

\subsection{Indústria 4.0}

As revoluções industriais ocorridas permitem diversas e significativas mudanças para as organizações e para a sociedade, onde dois aspectos se destacam neste contexto de transformação: a de tecnologia e a oganização social envolvida no processo $(\mathrm{Xu}, \mathrm{Xu}, \& \mathrm{Li}, 2018)$. O termo Indústria 4.0 foi primeiro utilizado na visão estratégica para a promoção de alta tecnologia da Alemanha. O tema também é bastante difundido como: Manufatura Avançada, Indústria Inteligente, e Internet das Coisas. (Cavalcante; Almeida, 2017).

Com o advento da internet e de tecnologia de controle específicas denominadas sistemas ciber-físicos, haverá uma transformação profunda das organizações e também das relações trabalhistas e produtivas, além das relações com clientes e 
fornecedores e sociedade de uma forma geral (Schwab, 2018).

Esta realidade industrial procura, por meio da integração de novos recursos tecnológicos de interação entre máquinamáquina e máquina-operador, onde otimizar os processos produtivos tornando-os eficientes e eficazes, possibilitando assim uma melhor gestão dos recursos tecnológicos e humano nas organizações e consequentemente para um crescimento da própria economia (de Oliveira Morais et. al, 2018). Segundo Costa et al. (2013), a indústria 4.0 é a representação da atual tendência das tecnologias de automação na indústria de transformação e inclui principalmente os sistemas ciber-físicos (CPS), Internet das coisas (IoT) e computação em nuvem(CC). A Figura 1 representa as quatro revoluções industriais.

Figura 1: As revoluções industriais.

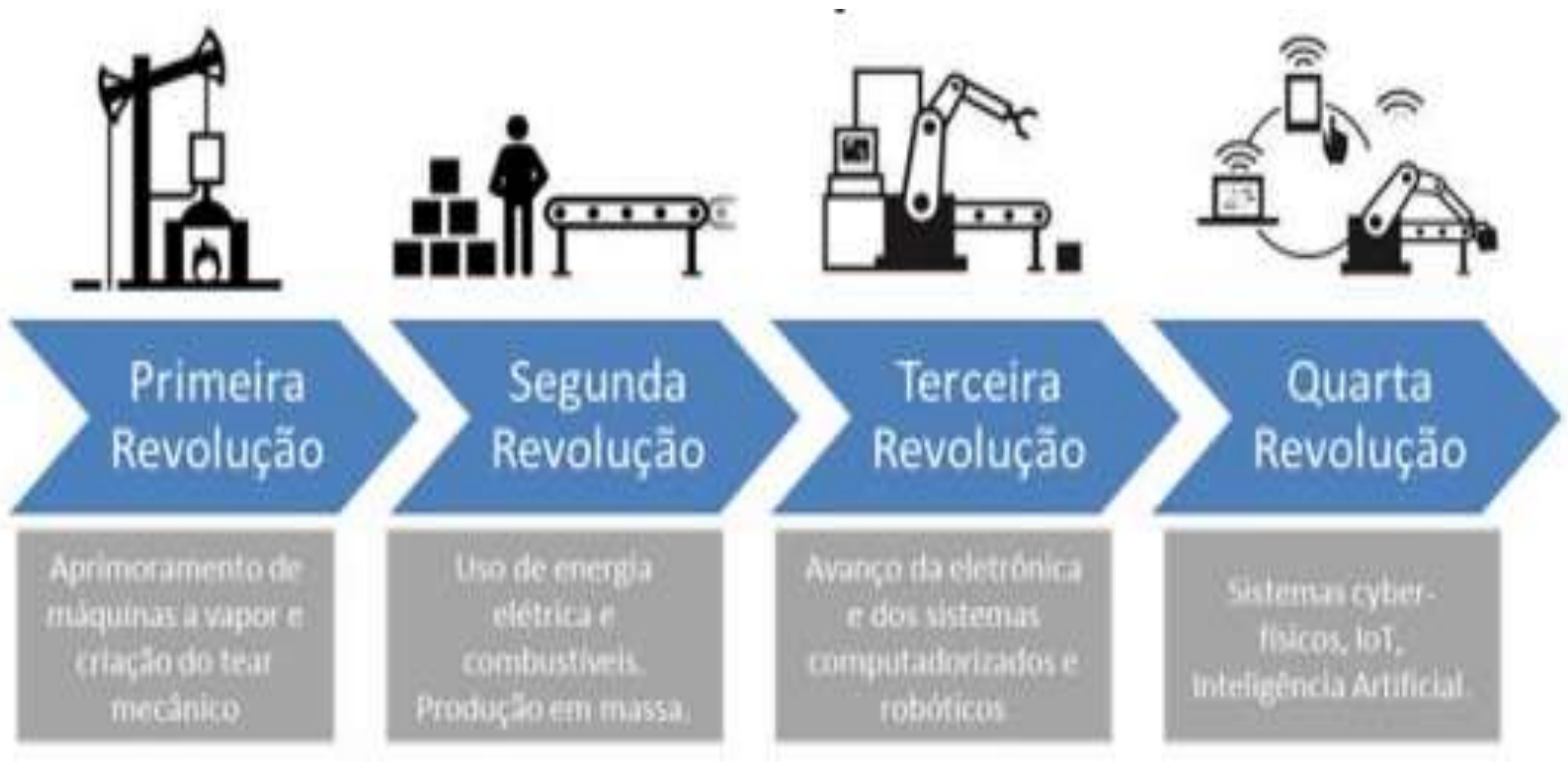

Fonte: Adaptado de Firmo (2020).

A Indústria 4.0 modifica não somente os sistemas produtivos, mas também todas as relações entre a sociedade e empresas em geral. Conhecida também como a revolução da internet, esse conjunto de tecnologias está transformando a integração entre máquinas, logística e suprimentos (Faria et al., 2017; Schwab, 2018).

Os sistemas produtivos se tornarão altamente flexíveis devido a uma produção mais individualizada. A cadeia de suprimentos deverá possuir configurações, tempos e rotas flexíveis. Os níveis de estoque e o setup de processo deverão continuar baixos para que os processos continuem ágeis (Faria et al., 2017).

Portanto, a Indústria 4.0 é um modelo de produção integrada virtualmente e habilitada a partir de tecnologias inteligentes interoperáveis. Dito de outro modo, são processos produtivos equipados com arquiteturas inteligentes que partindo de operações físicas ou ambientes virtuais, conformam produtos, serviços ou quaisquer outros entregáveis de produção (de Souza Silva Junior et. al, 2020).

As tecnologias da indústria 4.0 são de cunho orientativo e de apoio geral às empresas que queiram implantá-la, de forma a otimizar a produção de diferentes formas. Cada pilar tem suas estruturas e suas premissas, e cada empresa deve avaliar as medidas que cabem dentro de suas percepções (Santos et al.,2018).

$\mathrm{O}$ intuito é que as fábricas se tornem inteligentes e consigam resolver pequenos problemas sem a ajuda de humanos, apenas com os sistemas conectados e a robotização (Schwab, 2018; Almada-lobo, 2015). Nas fábricas, o objetivo é reduzir o estoque e produzir produtos diferenciados segundo especificações dos consumidores. A figura 2 demonstra as tecnologias que permeiam a Indústria 4.0, conforme segue. 
Figura 2: Tecnologias que permeiam a I4.0.

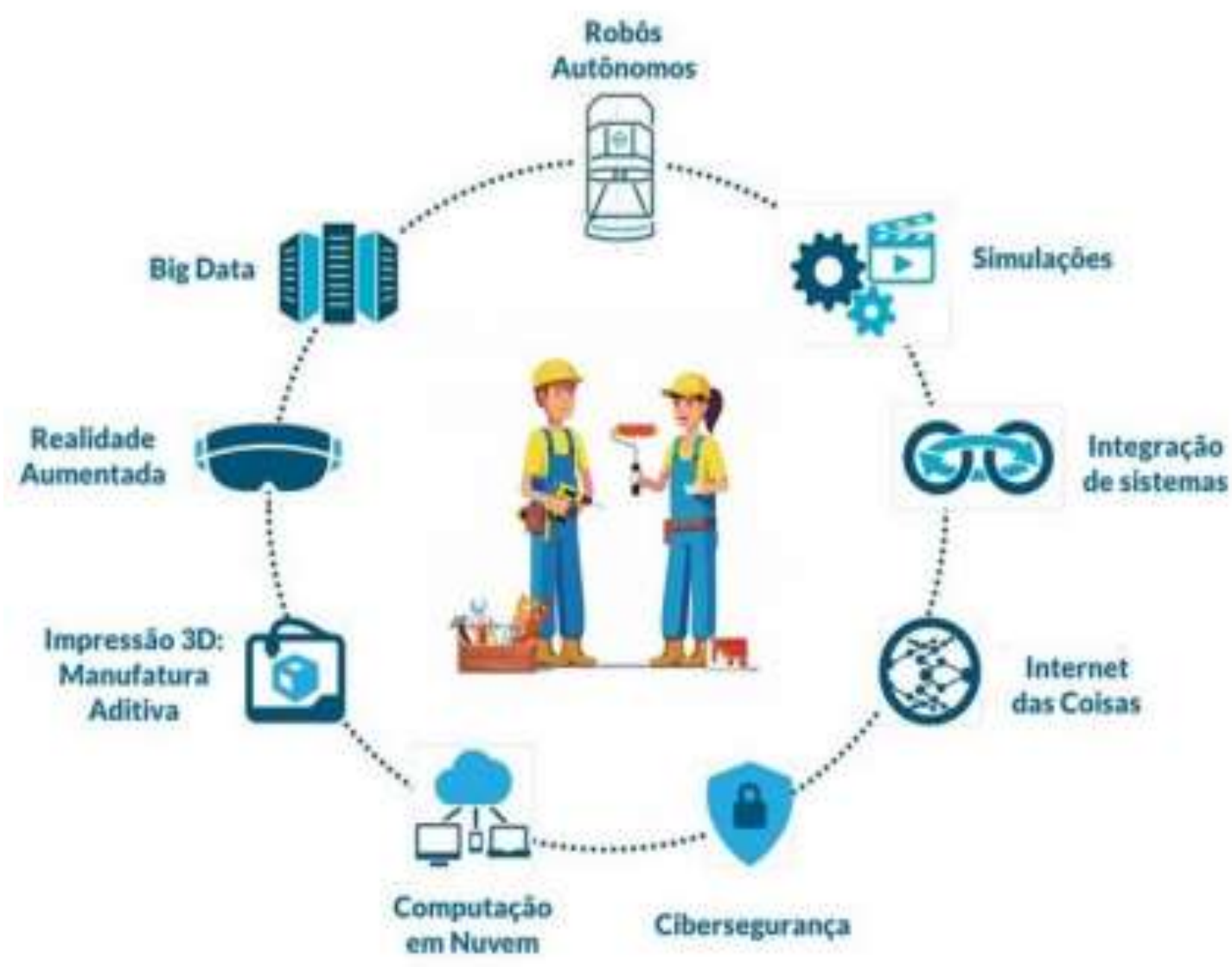

Fonte: Alves (2018).

$\checkmark$ Big Data: coletar e análise de grandes quantidades de dados permitindo otimizar e potencializar a qualidade da produção e melhorar os sistemas e os equipamentos.

Robôs Autônomos: utilização pela indústria de robôs para lidar com tarefas complexas, obtençãode mais autônoma e flexível possibilitando interagir com outros robôs e trabalham lado a lado com os seres humanos com segurança, aprendendo com eles.

$\checkmark$ Simulação pode ser feita por meio de softwares específicos. Eles captam os dados da produção e fazem análises de variáveis e de intervalos de tempo. Juntos, os indicadores conseguem apontar onde estão os gargalos da produção e o que poderia ser feito para solucioná-los. A efetividade da simulação depende da quantidade e qualidade dos dados que alimentam os sistemas. Por isso, para implementar esta metodologia, a fábrica precisa já ter uma estrutura de dados organizada, que os unifique e padronizem, a fim do sistema poder usar as informações.

$\checkmark$ Integração de Sistemas: horizontal e vertical: a maioria dos sistemas de TI (tecnologiada informação) atualmente não estão totalmente integrados. No entanto, com a Indústria 4.0 empresas, departamentos, funções e capacidades, será muito mais coesa. Redes de integração de dados universais permitirão que as cadeias de valor sejam verdadeiramente automatizadas. 
Internet Industrial de Coisas: refere-se à extensão e uso da internet das coisas (IoT) em setores industriais e aplicações. Com um forte foco na comunicação máquina a máquina, big data e aprendizado de máquina, ela permite que indústrias e empresas tenham mais eficiência e confiabilidade em suas operações.

$\checkmark$ Cibersegurança: é a prática que protege computadores e servidores, dispositivos móveis, sistemas eletrônicos, redes e dados contra ataques maliciosos. Também é chamada de segurança da tecnologia da informação ou segurança de informações eletrônicas.

$\checkmark$ Computação em Nuvem: permite registrar um maior número de tarefas relacionadas a produção requer um maior intercâmbio de dados entre os locais e empresas. disponibilidade sob demanda de recursos do sistema de computador, especialmente armazenamento de dados e capacidade de computação, sem o gerenciamento ativo direto do utilizado.

$\checkmark$ Fabricação Aditiva: é o processo de criar objetos sólidos tridimensionais a partir de modelos digitais. Impressão 3D é alcançado com o uso de processos aditivos, quando um objeto é criado pela sobreposição de diversas camadas de material. A impressão 3D é considerada diferente das técnicas tradicionais, como o processo substrativo, que se baseia na remoção de material por corte ou perfuração.

$\checkmark$ Realidade Aumentada: tem a finalidade de suportar uma variedade de serviços, bem como a seleção de peças em um determinado armazém e de reparação do transporte por meio de dispositivos móveis. Estes sistemas estão em seus estágios iniciais, porém no futuro as empresas vão fazer uma realidade aumentada mais ampla favorecendo aos trabalhadores informações em tempo real, com isso melhorando a tomada de decisões.

As indústrias estão cada vez mais propensas a utilização da digitalização e integração das cadeias verticais e horizontais, assim como a digitalização de serviços e produtos oferecidos, transformando o modelo de negócio atual em um modelo totalmente digital de maior e mais fácil acesso aos clientes, fornecedores, colaboradores e a sociedade como um todo, sendo a maneira para a obtenção de aspectos relevantes na transformação das tecnologias digitais na obtenção de uma maior agilidade nas organizações (de Oliveira Morais et. al, 2018).

Segundo Vita (2018), a Indústria 4.0 reúne os avanços tecnológicos contemporâneos que integram o mundo físico, com seus modelos virtuais, serviços e, finalmente, coordenação dentro de uma organização para criar uma cadeia de valor ágil e interconectada. Ainda segundo Klaus Schwab (2018), as inovações da indústria 4.0 se configuram mesmo com o aspecto de uma nova revolução e para sustentar sua convicção, ele apresenta três elementos que diferencia das revoluções anteriores: velocidade, amplitude e complexidade.

\subsection{Cultura da Inovação na Indústria 4.0}

O desempenho organizacional deriva da opção estratégica dos principais dirigentes organizacionais (Hayes \& Wheelwright, 1984). Uma opção estratégica conforme Schumpeter (1985) é a de maiores lucros com maior produção e menores esforços, induzindo ao que o autor chama de "inovação de mercado", ou seja, a conquista de clientes oferecendo produtos com preços menores. Estas ações repercutem em toda a vida organizacional, exigindo mudanças inclusive na estrutura social existente.

Para Ahmed (1998) as organizações mais inovadoras são as que possuem uma cultura voltada ao desenvolvimento da 
criatividade e que possibilitam aos atores organizacionais desenvolverem sua capacidade para inovar. As empresas estão caminhando para construção de uma inovação de maneira profunda e sustentável (Gibson, 2011), no entanto, essa construção muitas vezes não dá certo, não por falta de tentativa, mas porque a organização não possui as bases necessárias para que a cultura de inovação sobreviva (Wunker, 2015).

A cultura da inovação na industria 4.0 é vista como uma ferramenta estratégica para a produtividade e para o sucesso das empresas, comunidades e mesmo de um país. Como um conceito, a cultura da inovação é, cada vez mais, considerada um fenômeno multidimensional (não linear), que envolve gestão, processos e mercado, permitindo a geração de valor tangível e intangível (Green, 2015).

Empresas de pequeno e médio porte sentem ainda mais dificuldade para adequar-se à Indústria 4.0, pela complexidade das atividades envolvidas e pela necessidade de investimentos em todas as áreas da empresa. A Indústria 4.0 necessita de investimentos de modo que empresas que desejam ajustar-se a esse modelo nem sempre conseguem alcançar os resultados esperados em curto prazo (Souza e Gasparetto, 2018).

A cultura para a inovação permite à empresa ser mais flexível e a se reorganizar com mais agilidade caso haja uma adversidade, ou mesmo problemas comerciais, uma vez que as pessoas estão envolvidas nos processos independente de sua posição hierárquica na organização.

Segundo Terra (2012), existe uma busca veloz por diferenciação no mercado mundial, que se fazem necessárias estruturas flexíveis que favoreçam as competências globais e aprimorem as criações de forma personalizada as necessidades dos clientes.

A Figura 3 exemplifica os fatores que envolvem a cultura da inovação relacionados ao processo organizacional.

Figura 3: Cultura da Inovação.

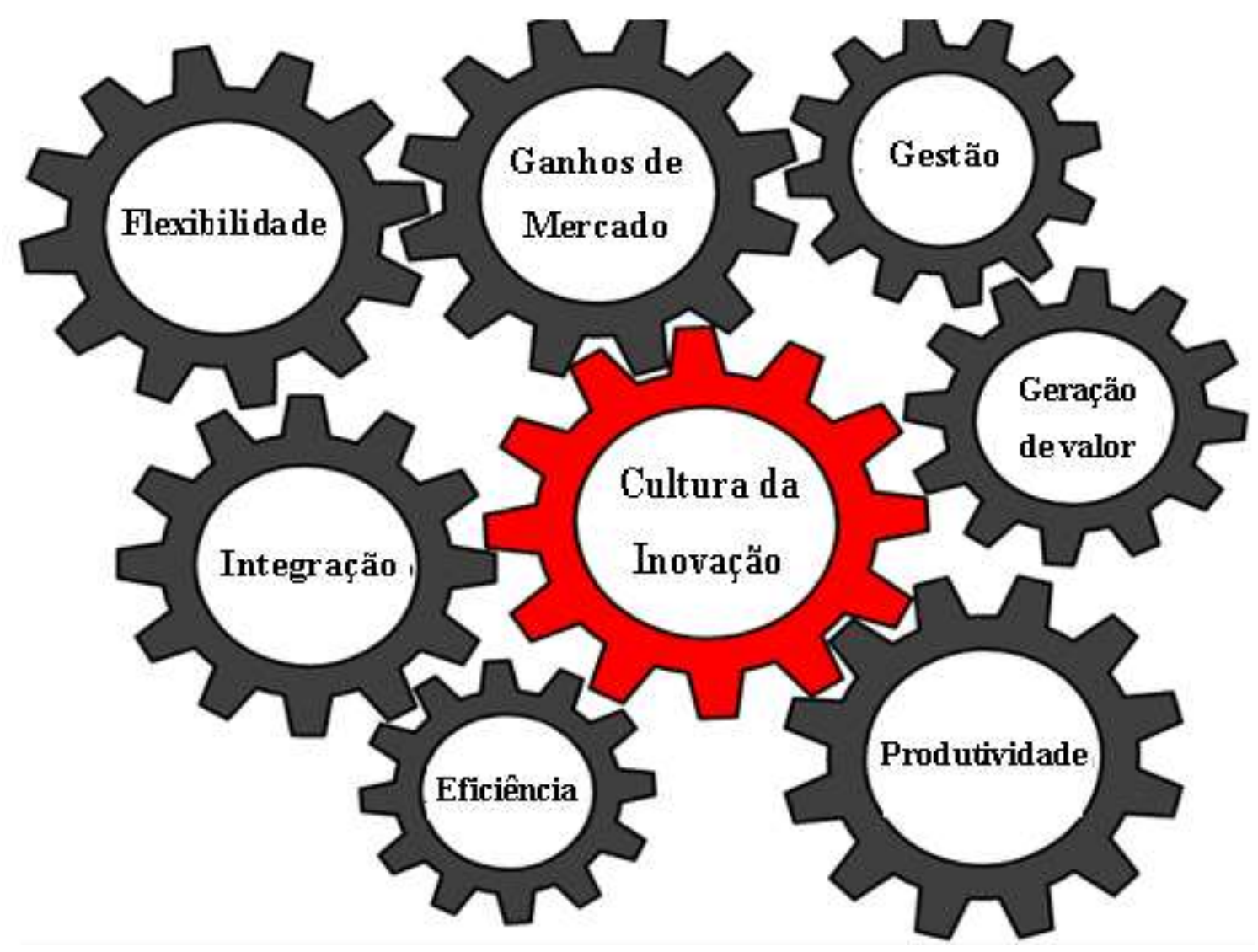

Fonte: de Oliveira Morais et. al, (2018). 
Elementos importantes compõem a cultura da inovação tais como: Flexibilidade, integração, eficiência, produtividade, geração de valor, gestão e ganhos de mercado promovem uma sinergia potencializando a organização permitindo um crescimento e fomentando um ambiente favorável ao processo de inovação, eliminando alguns possíveis entraves com os envolvidos (de Oliveira Morais et. al, 2018).

\section{Metodologia}

Nesta seção, serão descritos os procedimentos metodológicos que nortearam o desenvolvimento deste trabalho. Neste estudo, no que se refere aos objetivos, a pesquisa possui características de referenciais teóricos. A revisão sistemática da literatura é caracterizada pela busca rigorosa de pesquisas relacionadas com seus termos de busca que seguem um protocolo e análise dos estudos localizados (Ferenhof; Fernandes, 2016).

Além disso, quanto à natureza, o presente estudo é uma investigação básica, cuja finalidade é gerar novos conhecimentos para avançar o tema "Indústria 4.0" sem aplicação prática prevista, para um problema específico.

Segundo Marconi e Lakatos (2009), essa abordagem tem como preceito investigar e desvendar aspectos mais intrínsecos: "permitindo descrever detalhadamente investigações, atitudes e tendências de comportamento, buscando interpretar e compreender as relações acerca da complexidade do problema sem o uso de recursos estatísticos".

\section{Resultados e Discussão}

Conforme exposto no referencial teórico aliar inovação organizacional, elementos da industria 4.0 e cultura da inovação passa a ser um importânte tripé a ser considerado quando se trata de elementos que favoreçam as práticas relacionadas a melhorias de desempenho e em especias a ampliação e potencialização da competitividade organizacional na visão dos autores deste artigo.

Uma empresa possui certa vantagem competitiva quando, segundo Barney \& Hesterly (2006), torna-se capaz de gerar um mais valor econômico do que os seus concorrentes sejam estes diretos ou mesmo indiretos. Sendo assim, as inovações são a principal iniciativa que uma organização tem como proposta de garantir esse diferencial. As organizações transnacionais da era do mercado globalizado têm como principal diferencial competitivo a inovação de produtos ou serviços, para se adaptarem aos novos mercados.

Essas vantagens vão além de sua aplicação físicas e passam a ser incorporadas na cultura organizacional, tornando-se cada vez mais relevantes no dia a dia da empresa, principalmente, através do uso gerencial para a tomada de decisão mais acertiva.

As transformações organizacionais são um processo necessário, complexo e irrevercível que deve ser absorvido de forma gradativa permitindo assim um conhecimento enraizado seja na organização como também pelas pessoas envolvidas no processo.

Segundo Costa et al. (2011), organizações de todos os lugares estão enfrentando crescente pressão para transformar modelos de negócios centrados no produto em novos modelos focados na criação e captura de diferentes fontes de valor.

Entre os principais focos dessa estratégia competitiva estão as melhorias nos processos de produção através do avanço da arquitetura de sistemas, da interoperabilidade, da produção customizada, dentre outros fatores propulsores para as organizações (Kagermann et al., 2016). Os gestores cada vez mais buscam meios de compartilhamento de conhecimento bem como novas formas de desenvolve-lás, fazendo com que surjam novos modelos a serem aplicados e discutidos quanto a sua finalidade onde no ambiente organizacional a relação entre as pessoas e as organizações devem ser desenvolvidas, ampliadas e mantidas, visando o fortalecimento das organizações proporcionando uma maior competitividade e lucratividade, 
As aplicação das tecnologias imergentes assim como a gestão da inovação e do conhecimento são engrenagens que fazem com que as empresas se movimentem, e para isto agregar novas situações e ferramentas gerenciais deve sempre ser tratada com seriedade e prioridade, pois também é considerada um fator diferencial nos sucessos das empresas que a utilizam.

Ampliar as vantagens competitivas por meio de novas ferramentas gerenciais torna o processo viável, seja para quem propõe ou mesmo para aqueles que estão no processo de execução direta desta novas diretrizes e portanto podem ter algum tipo de resistência quanto ao novo. Buscar desenvolver este processo eliminando possíveis barreiras passa a ser um grande desafio no processo de gestão.

A Figura 4 apresenta, segundo os autores pode ser um caminho para o entendimento de tais ações junto aos envolvidos.

Figura 4: Tripé para uma organização competitiva.

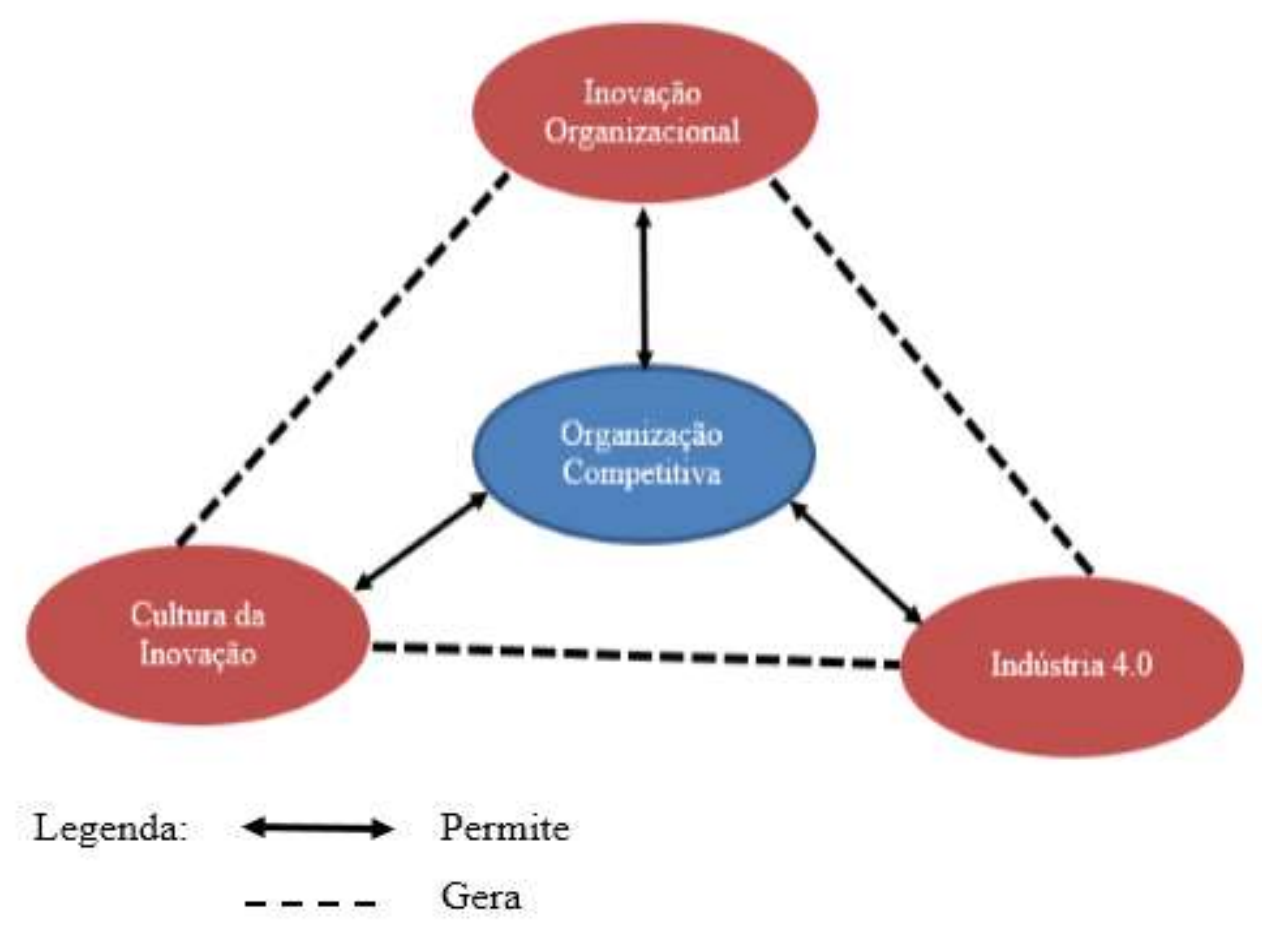

Fonte: Autores.

Sem a pretençao de esgotar as possibilidades e sim de fomentar tais aplicações os autores sugerem o tripé apresentado na Figura 4 como um dos elementos norteadores para que as organizações possam aplicar e tornar-se cada vez mais competitivas, levando em consideração todo o processo de inovação organizacional, cultural organizacional e da gestão a Indústria 4.0, são interfaces importantes dentro e fora das organizações, entre clientes e fornecedores.

A Figura 4 apresentada sugere que para a organização ser competitiva este deve permitir que haja uma interação entre a indústria 4.0 por meio de uma cultura da inovação onde estas sejam compartilhadas em toda a empresa e que também seja possível gerar interfaces entre estes elementos promovendo assim a sua sustentabilidade no que se refere as questões de competitividade.

Considerando que cada vez mais as questões de competitividade estará presente nas organizações gerar novas metodologias passa a ser de extrema relevância, independentemente de sua complexidade, a empresa deve buscar ser flexível para atender as novas demandas. Seguindo esse contexto, elaborar metodologias alternativas está entre os principais fatores de competitividade de uma organização (Lau, 2015). 


\section{Conclusão}

Elaborar estratégias para o aumento da competitividade da empresa torna o gerenciamento para as novas demandas mais eficiente e eficaz na obtenção dos resultados esperados, bem como no atendimento e as espectativas do mercado em que a empresa atua e consequentemente pelos seus clientes.

Ainda considerando os aspectos teóricos relativos abordados aos ganhos de vantagem competitiva estas podem ser associadas a capacidade de inovar em processos, serviços e produtos. Temos ainda que as revoluções tecnológicas, que levaram a indústria ao status de Indústria 4.0, também pesam por transformações e adequações cada vez mais frequentes onde compreender ou mesmo analisar tais elementos faz com que se obtenha um alto patamar de competitividade nas organizações.

Levando-se em consideração da concepção de vantagem competitiva, entende-se que os recursos e capacidades e o ambiente competitivo em que se insere podem ser um facilitador quanto a estratégia adotada pela empresa, a qual afeta o seu desempenho onde a forma de trabalho vem modificando a gestão de organizacional. Esse novo modelo de gestão exige tecnologias e modelos inovadoras para solucionar problemas complexos de maneira imediata e acertada.

O processo de inovação passa a ter maior visibilidade uma vez que tanto os produtos, processos e/ou serviços estão sendo exigidos com maior frequencia o que impacta diretamente toda a cadeia produtiva desde o fornecedor até o cliente final, por este motivo promover ações para a busca de uma maior competitividade permite com que as organizações possa se destacar no mercado em que atuam.

Elaborar, criar e utilizar novos conhecimentos gera possibilidades cada vez mais diversas o que potencializa as futuras ações a serem criadas independentemente do porte ou segmento da organização, oferecer benefícios e desafiar os processos existentes passa a ser o desafio do processo de gestão.

\subsection{Trabalhos futuros}

$\checkmark$ Aplicação do modelo proposto e analisar quais os seus resultados nas organizações.

\section{Agradecimentos}

$\checkmark$ Aos nossos familiares pelo apoio, carinho e compreensão.

\section{Referências}

Ahmed, P. K. (1998). Culture and climate for innovation. European journal of innovation management.

Almada-Lobo, F. (2015). The Industry 4.0 revolution and the future of Manufacturing Execution Systems (MES). Journal of innovation management, 3(4), $16-21$.

Barney, J. B., \& Hesterly, W. S. (2006). Strategic management and competitive advantage. Upper Saddle River, N.J.: Pearson/Prentice Hall.

Cavalcante, C. G. S., \& Almeida, T. D. D. (2018). Os benefícios da Indústria 4.0 no gerenciamento das empresas. Journal of lean systems, 3(1), 125-152.

Costa, D. D. M., Barbosa, F. V., \& Silva, C. H. P. D. (2011). Empreendedorismo e inovação: o papel da educação superior nas economias mundiais.

Costa, S., Borini, F. M., \& Amatucci, M. (2013). Inovação global de subsidiárias estrangeiras localizadas em mercados emergentes. Revista de Administração Contemporânea, 17, 459-478.

de Oliveira Morais, M., de Araujo, N. B., dos Santos Aleixo, H. W., \& de Oliveira Uchoa, L. S. (2018). Conhecimento e o capital humano na indústria 4.0. Brazilian Journal of Development, 4(7), 4570-4583.

de Souza Silva Junior, D., Carlos dos Santos, R., \& Luiz dos Santos, I. (2020). Inovações Da Indústria 4.0 Na Gestão De Processos Na Prestação De Serviços Na Construção Civil. Future Studies Research Journal: Trends \& Strategies, 12(3).

Gibson, R. (2010). Excelência em inovação: lições das campeãs brasileiras. Harvard Business Review, 85(2), 129-138.

Hayes, R. H., Hayes, R. H., \& Wheelwright, S. C. (1984). Restoring our competitive edge: competing through manufacturing (Vol. 10). John Wiley \& Sons Incorporated. 
Magalhães, D. (2020). Uma Visão Geral Sobre Processo De Desenvolvimento De Produtos, Inovação, Gestão Do Conhecimento, Startup E Indústria 4.0. Enciclopédia Biosfera, 17(33).

Ferenhof, H. A., Fernandes, R. F. (2016). Desmistificando a revisão de literatura como base para redação científica: método SFF. Revista ACB, $21,550-563$.

Firmo, A. 5G e a Indústria 4.0, (2020). Disponível em: Enciclopédia Biosfera, Centro Científico Conhecer. 17, $4062020<$ https://medium.com/embeddedufcg/5g-e-a-ind\%C3\%BAstria-4-0-2601ddeb27c9>. Acesso em: 04 mar. 2021.

Green, R., Agarwal, R., Brown, P. J., Bajada, C., \& Shashnov, M. (2015). Innovation Capability and the Food Beverage and agri-business sectors.

Hofmann, E., \& Rüsch, M. (2017). Indústria 4.0 e o status atual, bem como as perspectivas futuras em logística. Computadores em Indústria, 89, $23-34$.

Kagermann, H., Anderl, R., Gausemeier, J., Schuh, G., \& Wahlster, W. (Eds.). (2016). Industrie 4.0 in a Global Context: strategies for cooperating with international partners. Herbert Utz Verlag.

Kamble, S. S., Gunasekaran, A. E., \& Gawankar, S. A. (2018). Estrutura da indústria sustentável 4.0: uma revisão sistemática da literatura identificar as tendências atuais e perspectivas futuras. Segurança de Processo e Proteção Ambiental, 117, 408-425.

Lau, A. K., \& Lo, W. (2015). Regional innovation system, absorptive capacity and innovation performance: An empirical study. Technological Forecasting and Social Change, 92, 99-114.

Low, J., \& Kalafut, P. C. (2003). Vantagem invisivel: como os intangiveis conduzem o desempenho da empresa. Bookman.

Organisation Economic co-operation and Development OCDE (2005). Education at a glance: OCDE indicators. OCDE.

Rasheed, A., San, O., \& Kvamsdal, T. (2020). Digital twin: Values, challenges and enablers from a modeling perspective. Ieee Access, 8, $21980-22012$.

Rubmann, M., Lorenz, M., Gerbert, P., Waldner, M., Justus, J., Engel, P., \& Harnisch, M. (2015). Industry 4.0. Boston Consulting Group

Santos, B. P., Alberto, A., Lima, T. D. F. M., \& Charrua-Santos, F. M. B. (2018). Indústria 4.0: desafios e oportunidades. Revista Produção e Desenvolvimento, 4(1), 111-124.

Santos, D. F. L., Basso, L. F. C., Kimura, H., \& Kayo, E. K. (2014). Innovation efforts and performances of Brazilian firms. Journal of Business Research, 67(4), 527-535.

Santos, D. F. L., Basso, L. F. C., \& Kimura, H. (2012). A estrutura da capacidade de inovar das empresas brasileiras: uma proposta de construto. RAI Revista de Administração e Inovação, 9(3), 103-128.

Souza, E. S. D. (2018). Características e impactos da indústria 4.0: percepção de estudantes de ciências contábeis.

Schumpeter, J. A. T. D. D. (1982). Econômico. Abril Cultural.

Schumpeter, J. A. (1985). O fenômeno fundamental do desenvolvimento econômico. A teoria do desenvolvimento econômico. Nova Cultural.

Schwab, K. (2019). A quarta revolução industrial. Edipro.

TERRA, J. C. (2012). Dimensões da gestão da gestão de inovação: uma abordagem para transformação organizacional.

Vita, RO (2018). Integração da Indústria 4.0 e Manufatura Enxuta e o Impacto no Desempenho Organizacional.

Wunker, S., \& Farber, D. (5). Strategies big businesses use to build a culture of innovation. Forbes. July, 29, 2015.

Xu, L., Xu, E., \& Li, L. (2018). Indústria 4.0: estado da arte e tendências futuras. International Journal of Production Research, 56(8), $2941-2962$.

Zhou, K., Liu, T., \& Zhou, L. (2015, August). Industry 4.0: Towards future industrial opportunities and challenges. In 2015 12th International conference on fuzzy systems and knowledge discovery (FSKD) (pp. 2147-2152). IEEE. 